\title{
Toosendanin Suppresses Glioma Progression Property and Induces Apoptosis by Regulating miR-608/Notch Axis
}

This article was published in the following Dove Press journal: Cancer Management and Research

\author{
Qiong Wang ${ }^{1-3}$ \\ Zeng Wang ${ }^{1-3}$ \\ Guilan Hou ${ }^{1-3}$ \\ Ping Huang ${ }^{1-3}$
}

'Department of Pharmacy, Institute of Cancer and Basic Medical Sciences of Chinese Academy of Sciences, Hangzhou City, Zhejiang Province 310022, People's Republic of China; ${ }^{2}$ Department of Pharmacy, Cancer Hospital of the University of Chinese Academy of Sciences, Hangzhou City, Zhejiang Province 310022, People's Republic of China; ${ }^{3}$ The Department of Pharmacy, Zhejiang Cancer Hospital, Hangzhou City, Zhejiang Province 310022, People's Republic of China
Correspondence: Ping Huang The Department of Pharmacy, Zhejiang Cancer Hospital, No. I Guangji East Road, Gongshu District, Hangzhou City, Zhejiang Province, People's Republic of China,

Tel +86057I-88I22425

Email PingHuangfjk@163.com
Background: Glioma is one the most common and aggressive primary tumors of adult central nervous system worldwide, which tends to develop dysplasia and metastasis. Recently, toosendanin (TSN) has shown pharmacological effects in several cancers. However, little is known about the underlying mechanism of the effect of TSN on glioma and its relationship between miRNA in glioma.

Methods: Cell proliferation, cell cycle, cell apoptosis and cell migration were analyzed by CCK-8 cell viability, flow cytometry, wound scratch healing, transwell and Western blotting assays, respectively, in vitro. The regulation relationships between TSN and miR-608 or between miR608 and Notch1 (Notch2) were examined using qRT-PCR, dual-luciferase and Western blotting assays. The functional effects of TSN through regulating miR-608 and Notch1 (Notch2) were further examined using a xenograft tumor mouse model in vivo.

Results: After TSN concentration was increased from $50 \mathrm{nM}, 100 \mathrm{nM}$ to $150 \mathrm{nM}$, cell proliferation and cell cycle were gradually reduced, and the cell apoptosis rate was increased in U-138MG or U-251MG cells. Wound-healing and transwell assays results showed that cell migration was significantly inhibited in TSN treatment cells (TSN treatment, $50 \mathrm{nM}$ ) compared to control cells. Mechanistic studies revealed that TSN up-regulated the expression of microRNA-608 (miR-608), while down-regulated the expression of miR-608's target, Notch1 and Notch2. Over-expression of Notch1 and Notch2 partly attenuated TSN-induced tumor suppressive function. Moreover, in vivo experiments revealed that TSN treatment led to a significant inhibition of tumor growth, suggesting that it might be a promising drug for the treatment of glioma.

Conclusion: In the present study, a novel established functional manner of TSN/miR-608/ Notch1 (Notch2) axis was systematically indicated, which might provide prospective intervention ways for glioma therapy.

Keywords: glioma, TSN, miR-608, Notch1 and Notch2, tumor growth, metastasis

\section{Introduction}

Glioma is the most common and aggressive primary tumor in adult central nervous system (CNS) worldwide, accounting for approximately $30 \%$ of CNS tumors and $80 \%$ of brain malignancies. ${ }^{1,2}$ With an incidence rate of 3.2 per 100,000 population, glioma can be classified into high-grade (grade 3 and grade 4) and low-grade (grade 1 and grade 2) according to World Health Organization (WHO). ${ }^{3}$ With a median survival time of 11.6 years, $47 \%$ of glioma patients in low-grade glioma have a 10 -year survival rate. ${ }^{4}$ For highgrade glioma patients, the median overall survival time of grade 3 patients is 
approximately 3 years, and grade 4 glioma patients only stand at about 15 months. ${ }^{5}$ Although treatment strategies, including surgical resection, radiotherapy and chemotherapy, have been improved, the prognosis of glioma patients is still poor, mainly due to the malignant hyperplasia and invasion. ${ }^{6}$ Therefore, elucidating the molecular mechanism of glioma proliferation and invasion is an urgent need to identify effective therapeutic targets.

Recently, natural products are reported to play a critical role in the research and discovery of antitumor drugs. Bitter melon extract was found to induce p62 accumulation and autophagic cell death by modulating AMPK/mTOR signaling pathway in breast cancer. ${ }^{7}$ In addition, the effects of bitter melon extract on cell proliferation and the regulatory T cell (Treg) population in head and neck squamous cell carcinoma (HNSCC) were demonstrated. ${ }^{8,9}$ As a triterpenoid saponin from the bark of the trees Melia toosendan and Mazedarach (Meliaceae), Toosendanin (TSN) exhibits anti-proliferative and apoptosisinducing effects on various human cancer cells in vitro, including hepatocellular carcinoma, prostate cancer, leukemia, and lymphoma. ${ }^{10}$ Zhang et al demonstrated that TSN acts as a novel inhibitor of signal transducer and activator of transcription 3 (STAT3), which blocks tumorigenesis in osteosarcoma. ${ }^{11}$ Pei et al showed that TSN inhibits pancreatic cancer progression via down-regulating Akt/mTOR signaling. ${ }^{12}$ Additionally, TSN could be used as a novel PI3K inhibitor to reverse breast cancer resistance. ${ }^{13}$ However, little is known about TSN in Glioma. So far, existing findings just showed that TSN in glioma was involved in $\operatorname{Er} \beta$ up-regulation, p53 activation and further promotes cell apoptosis. ${ }^{14}$ The role of TSN in glioma and the underlying mechanism need further study.

microRNAs (miRNAs), one type of small noncoding RNAs with 18-22 nt in length, usually regulate tumor-related mRNAs and serve as tumor promotor or suppressors. ${ }^{15}$ For example, miR-203 expression is significantly higher in ERpositive breast cancer patients and anti-miR-203 suppresses tumor growth and stemness by targeting suppressor of cytokine signaling 3 (SOCS3). ${ }^{16}$ miR-18a has a promoting effect on glioma via inhibiting retinoic acid receptor-related orphan receptor A (RORA) and activating the TNF- $\alpha$ mediated NF$\kappa \mathrm{B}$ signaling pathway. ${ }^{17}$ Recent studies showed that the biological activity of TSN was related to miRNAs. TSN was reported to inhibit the human oncogenic phenotype of gastric cancer via miR-200a/ $\beta$-catenin axis. ${ }^{18}$ However, whether TSN involves in miRNA-mediated anti-tumor affect in glioma remains unknown. Increasing evidence have indicated that miR-608 exerts important functions in the development of cancers. He et al demonstrated that miR-608 could inhibit HCC cell proliferation possibly via targeting BET family protein BRD4. ${ }^{19}$ miR-608, along with miR-342-5p can target NAA10 and inhibit colon cancer tumorigenesis. ${ }^{20}$ Moreover, tumor-suppressive role of miR-608 has been found in lung adenocarcinoma $^{21}$ and bladder cancer. ${ }^{22}$ More interestingly, MiR-608 inhibits the migration and invasion of glioma stem cells by targeting macrophage migration inhibitory factor, suggesting that miR-608 may act as a potential tumor suppressor in glioma. ${ }^{23}$ However, whether the effect of TSN is related to miR-608 is worth further study.

Notch signaling plays an important oncogenic role in glioma. When nuclear translocation occurs, Notch1 could regulate other important genes, such as p53, which is closely associated with glioma progression. ${ }^{24}$ Notch2 has been identified as an important prognostic marker in glioma, which may be involved in cell proliferation and invasion. ${ }^{25}$ Some miRNAs have been found to be involved in tumor development by targeting Notch signaling members individually or collectively. Among the identified glioma-associated miRNAs, miR-34a could affect the cell cycle arrest and cell death by inhibiting the expressions of c-Met, Notch-1, Notch-2 and CDK6. $^{26}$ In addition, miRNA-326 partially mediated toxic effects on both established and stem cell-like glioma lines through knocking down Notch. ${ }^{27}$ These findings showed that blocking Notch signaling could suppress glioma progression. However, whether Notch-1 and Notch-2 expressions are affected by TSN-mediated miRNA dysregulation remains to be explored.

In the present study, we investigated the effect of TSN on glioma progression. The influences of TSN treatment on the proliferation, apoptosis and migration of glioma cells were studied. Regulation of miR-608/Notch1 (Notch2) axis might be a possible mechanism of TSN. Furthermore, the effects of Notch1 or Notch2 over-expression on TSN-caused cell changes in cellular behavior were analyzed, highlighting their potential as novel candidates for glioma therapy.

\section{Materials and Methods}

\section{Cell Culture}

Human glioma cell lines (U-138MG and U-251MG) were all obtained from American Type Culture Collection (ATCC, Rockville, MD). Human normal astrocytes (NHA) were obtained from ScienCell (San Diego, CA), and served as a normal counterpart of glioma. Cells were cultured in Dulbecco's Modified Eagle Medium supplemented with 10\% fetal bovine serum and penicillin/streptomycin (Thermo Fisher 
Scientific). These cells were cultured at $37^{\circ} \mathrm{C}$ in a humidified condition containing $5 \% \mathrm{CO}_{2}$.

\section{Cell Treatment}

TSN is a triterpene, extracted from the skin of the azalea fruit. Purified Toosendanin (MF: C30H38O11, MW: 574.62, purity: HPLC $\geq 98 \%$ ) was purchased from Shanghai Yuan Ye Biotechnology Co. Ltd (Shanghai, China). U-138MG or U-251MG cells were exposed to TSN, whose concentrations gradually increased from $0 \mathrm{nM}, 50 \mathrm{nM}, 100 \mathrm{nM}$ to $150 \mathrm{nM}$. After treatment, cells were collected for assays of cell proliferation, cell apoptosis and cell migration.

\section{Cell Proliferation Assay}

For cell viability curve assay, U-138MG or U-251MG cells $\left(6 \times 10^{3}\right.$ cells/well $)$ were, respectively, seeded onto 96-well plates and treated with TSN at different concentrations. To detect the viable cell numbers at $24 \mathrm{~h}$ and $48 \mathrm{~h}$, a Cell Counting Kit-8 (CCK-8, Dojindo, Tokyo, Japan) was used. A microplate spectrometer (Thermo Fisher Scientific) was employed to measure the absorbance $(450 \mathrm{~nm})$.

For colony formation assay, U-138MG or U-251MG cells $\left(1 \times 10^{3}\right)$ were first mixed into top agar $(1.5 \mathrm{~mL})$ and then added onto base agar. Colonies were stained by Crystal Violet after 3 weeks. And a dissection microscope (TE2000-U, Nikon, Japan) was used to count the colonies.

\section{Cell Cycle and Cell Apoptosis Assay}

Cell cycle and cell apoptosis assay were performed in $\mathrm{U}-138 \mathrm{MG}$ or U-251MG cells at 48 hours post-transfection. After fixed with $70 \%$ ethanol overnight, the cells were stained with propidium iodide (PI) (BD Biosciences, San Jose, CA) for cell cycle analysis. Double staining with propidium iodide (PI) and Annexin V (BD Biosciences, San Jose, CA) was used for cell apoptosis analysis. Then, a FACSCalibur system (BD Biosciences) was used for cell cycle and apoptosis analysis according to the manufacturer's instructions. To analyze cell apoptosis in tissues, a terminal deoxynucleotidyl transferase dUTP nick end labeling (TUNEL) BrightRed Apoptosis Detection Kit (BD Biosciences) was used according to the manufacturer's instructions. And a microscope (TE2000-U, Nikon, Japan) was used to count the TUNEL positive cells.

\section{Protein Extraction and Western Blotting}

RIPA lysis buffer (Beyotime Institute of Biotechnology, Nantong, China) was used to extract total proteins from the cells. For total protein concentration quantification, a BCA protein assay kit (Thermo Fisher Scientific, Rockford, IL,
USA) was used. Proteins were separated by sodium dodecyl sulfate-polyacrylamide gel electrophoresis (SDS-PAGE) and then electrophoretically transferred onto polyvinylidene fluoride (PVDF) membranes. The primary antibodies were used to incubate the membranes overnight at $4^{\circ} \mathrm{C}$, which were then incubated with a horseradish peroxidase (HRP)-conjugated anti-mouse/anti-rabbit IgG (Thermo Fisher Scientific). Antibodies included PCNA (1:1000), p21 (1:1000), CyclinD1 (1:1000), Cleaved caspase-3 (1:1000), Cleaved PARP (1:1000), E-cadherin (1:1000), N-cadherin (1:1000), Notch1 (1:1000), Notch2 (1:1000) and GAPDH (an internal control, 1:2000) and corresponding secondary antibodies (Goat anti-rabbit/mouse IgG, 1:2000) were used in the study, and the detailed information was presented in Table S1.

\section{Wound-Healing Assay}

For wound-healing assay, linear scratch wounds were made on the cell monolayer. Twenty-four hr post-scratch, cell migration images were taken using an inverted microscope (Nikon), of which the scrape distances were measured by means of image J software (http://rsb.info.nih.gov/ij/).

\section{Cell Invasion Assay}

For cell invasion assay, U-138MG or U-251MG cells $\left(1 \times 10^{5}\right)$ were seeded into the top chamber of an insert (Corning Costar Co., Cambridge, MA, USA). Cells were cultured in the top chamber with a serum-free medium. To attract cells moving to the lower chamber, a medium containing $10 \%$ FBS was used. Twelve hours later, the invaded cells underside of the chamber were imaged and counted after fixation and staining.

\section{RNA Extraction and Quantitative Real-Time PCR (qRT-PCR)}

Total RNA of cultured cells was isolated using the TRIzol reagent (Thermo Fisher Scientific, Rockford, IL, USA). Using a cDNA Reverse Transcription Kit (Thermo Fisher Scientific), cDNA was synthesized from total RNA. qRTPCR assay for miR-608 levels was performed by a SYBR PrimeScript miRNA RT-PCR Kit (Takara, Shiga, Japan). U6 was selected as endogenous control for miR-608 expression. Corresponding primer sequences were listed in Table S2.

\section{Plasmid Cell Transfection}

miR-608 mimics (miR-608), miR-608 inhibitors (miR-608 inh) or their corresponding controls (miR-NC or NC inh) were obtained from genepharma (shanghai, China). To insert the 3'-UTR of Notch1 and Notch2 into the pmiR- 
Glo dual-Luciferase reporter plasmid (Promega, Madison, WI, USA), a Cloning Kit (Vazyme Biotech, Nanjing, China) was used. The binding-site (CCACCCC) was mutated (GGUGGGG) and used as a control. According to the above methods, Notch1 and Notch2 expression plasmids containing their coding sequences (CDSs) were constructed. For cell transfection, a Lipofectamine 2000 transfection reagent (Thermo Fisher Scientific) was used. A GFP plasmid was used to determine the transfection efficiency, which is about $80 \%$. The primers for plasmid construct were presented in Table S3.

\section{Dual-Luciferase Reporter Assay}

Luciferase reporter assays were performed as previously reported. $^{28}$ When the cell confluence was at $70-80 \%, 1 \mu \mathrm{g}$ luciferase reporter plasmid and miR-608/miR-NC were cotransfected into U-138MG or U-251MG cells. The protein was extracted 24 hours after transfection. A Luciferase Reporter Assay System (Promega) was used for the luciferase activities test.

\section{Tumor Xenografts in Nude Mice}

To construct a mouse glioma xenograft model, thymic BALB/c nude mice (16-18g) and U-251MG cells were used. All experiments with nude mice were constructed in accordance with the Guidelines for the Care and Use of Laboratory Animals, which was presented by the National Institutes of Health ${ }^{29}$ and approved by the Ethics Committee of Zhejiang Cancer Hospital (Approval no.2019-05-014). The right flanks of mice (6 mice/group) were subcutaneously injected with stable U-251MG cells $\left(1 \times 10^{6}\right)$. Four days later, mice were randomly divided into two groups and received i.p. injection of TSN (1 mg/kg per 2 days, TSN group) and mice injected with DMSO (NC group) served as control. Tumor size was measured every week post-injection, and tumor volume was calculated by the formula: volume $\left(\right.$ glioma $\left.^{3}\right)=$ length $\times$ width $^{2} / 2$. The mice were sacrificed 4 weeks post-injection, and the corresponding tumors were isolated, photographed and weighted. Additionally, qRT-PCR analysis was conducted to determine the expression level of miR-608 of the tumor tissue from NC and TSN groups. IHC analysis was performed to detect the expression of Ki67, Notch1 and Notch2 in tumor tissues from NC and TSN groups.

\section{Immunohistochemistry (IHC) Assays}

Tissues were first fixed in paraformaldehyde and then embedded using paraffin. After that, sections $(5 \mu \mathrm{m})$ were cut. For IHC assay, the antigen was repaired after tissue sections were rehydrated. After that, antibodies for $\mathrm{Ki}-67$, Notch 1 and Notch 2 were used to incubate tissue sections overnight at $4{ }^{\circ} \mathrm{C}$. Then, corresponding secondary antibodies and 3 , 3'-diaminobenzidine (DAB) solution (Sigma-Aldrich, St. Louis, MO, USA) were used to visualize the sections.

\section{Statistical Analysis}

Data are presented as the mean \pm standard error of the mean (SEM). Student's $t$-test or one-way ANOVA was used to evaluate the statistical analysis. $P<0.05$ was considered statistical significant. */\#, $P<0.05 ; * * / \# \#, P<0.01$.

\section{Results}

\section{TSN Suppresses Glioma Cell Proliferation}

As a natural product, the chemical structure of TSN is shown in Figure 1A. To determine the influence of TSN on glioma, NHA, U-138MG and U-251MG cells were treated with TSN at different concentrations $(0 \mathrm{nM}, 50 \mathrm{nM}, 100 \mathrm{nM}$, 150nM). As shown in Figure 1B, the cell survival rate was not significantly changed in NHA cells after TSN treatment. Whereas, the cell survival rate was gradually reduced in U-138MG or U-251MG cells after TSN concentration was increased from 50nM to $150 \mathrm{nM}$. Similarly, the results of colony formation assay demonstrated that cell colony numbers were markedly decreased after TSN treatment compared to the control cells (Figure 1C). Cell cycle analysis revealed that TSN treatment led to an upregulated cell population in the G1-phase and a reduced cell population in the S-phase (Figure 1D). In addition, Western blot results showed that PCNA and CyclinD1 levels were significantly down-regulated, while p21 expression was increased after TSN treatment (Figure1E). These results demonstrated that TSN treatment could suppress cell proliferation in U-138MG or $\mathrm{U}-251 \mathrm{MG}$ cells.

\section{TSN Promotes Glioma Cell Apoptosis}

As shown in Figure 2A, TSN treatment promoted apoptosis in $\mathrm{U}-138 \mathrm{MG}$ or U-251MG cells. The apoptosis rate in U-138MG cells was up-regulated from $1.61 \%$ to $6.91 \%, 13 \%$ and $22 \%$, as the TSN concentration was increased from $0 \mathrm{nM}$ to $50 \mathrm{nM}$, $100 \mathrm{nM}$ and $150 \mathrm{nM}$. Accordingly, the apoptosis rate in U-251MG cells increased from $4.54 \%$ to $14.1 \%, 21.2 \%$ and $33.4 \%$. Furthermore, protein levels of cleaved caspase- 3 and cleaved caspase-PARP were significantly increased after TSN treatment, indicating its pro-apoptotic role (Figure 2B). 
A

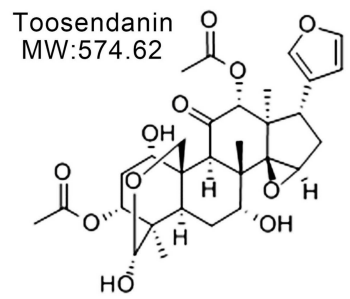

B

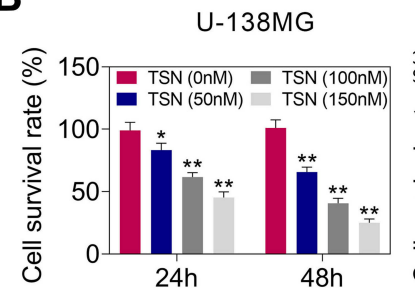

U-251MG

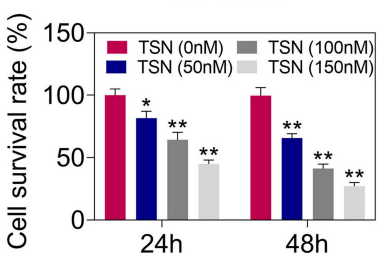

NHA

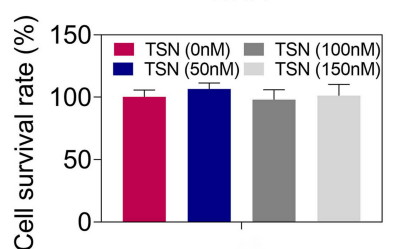

C

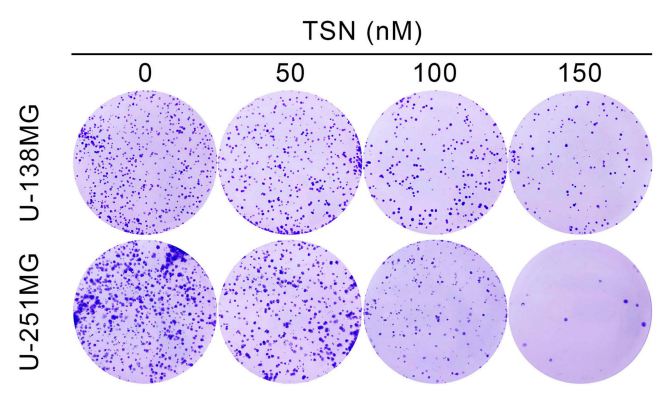

D
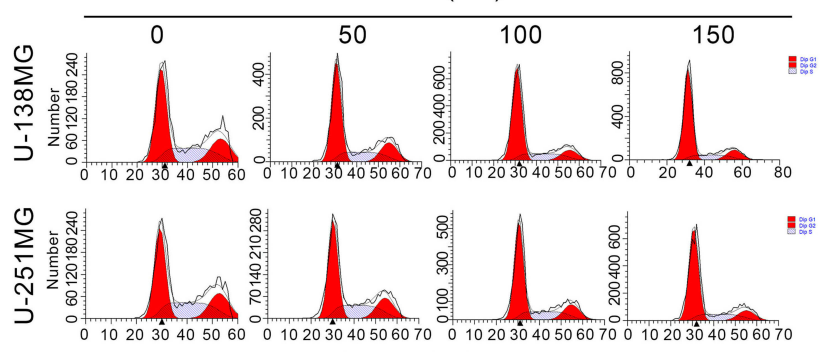
Channels (PE-H-PE-H)

$\mathbf{E}$
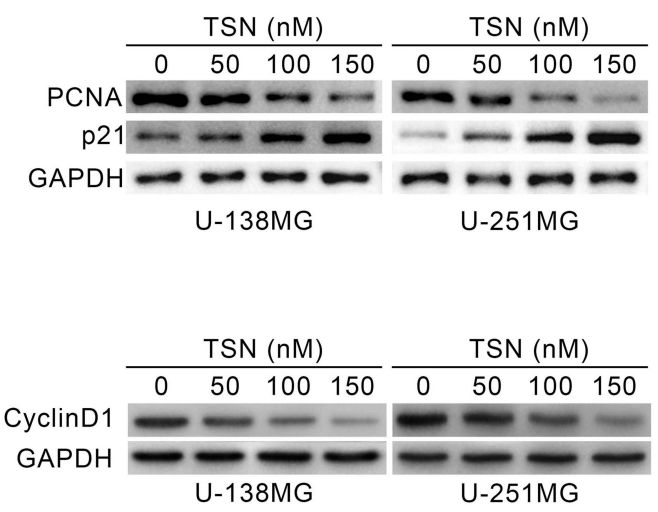

U-138MG

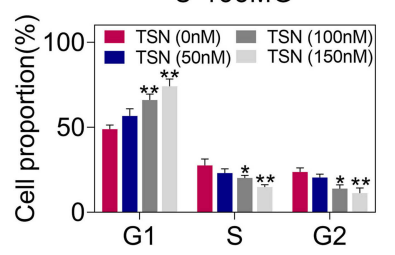

U-251MG

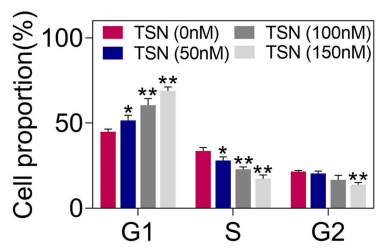

U-251MG

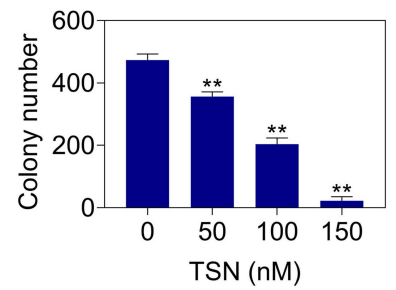

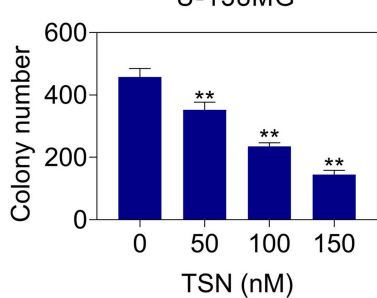

U-251MG

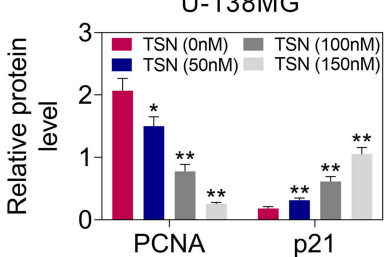

U-138MG

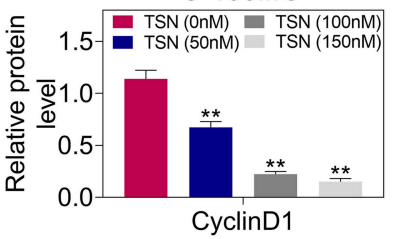

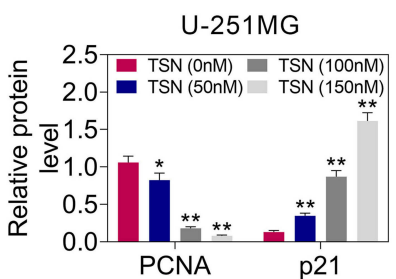

U-251MG

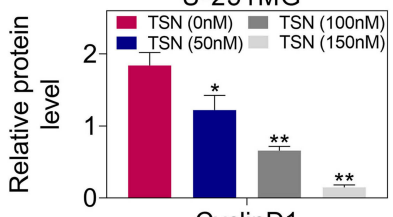

Figure I TSN affects the proliferation of glioma cells. (A) The chemical structure of TSN. (B) Cell survival of NHA, U-I38MG or U-25IMG cells at 24h and 48h after TSN treatment $(0 \mathrm{nM}, 50 \mathrm{nM}, 100 \mathrm{nM}, 150 \mathrm{nM})$. The measurements of the cell growth rate were obtained using a CCK-8 kit. (C) Colony formation analysis of U-I38MG or U-25IMG cells after TSN treatment (0nM, 50nM, I00nM, I50nM). Colony numbers were quantified and shown as histograms. (D) Cell cycle analysis of U-I38MG or U-25IMG cells after TSN treatment (0nM, 50nM, 100nM, I50nM). Cell percentage in GI, S and G2 phages were quantified and shown as histograms. (E) The protein levels of PCNA, P2I and CyclinDI in U-I38MG or U-25IMG cells after TSN treatment (0nM, 50nM, I00nM, I50nM), as determined using Western blotting. Relatively quantitative results were determined by Image $\mathrm{J}$ and shown as histogram. The data are expressed as the mean $\pm \mathrm{SEM}, * P<0.05, * * P<0.0 \mathrm{I}$.

\section{TSN Inhibits Glioma Cell Migration}

Wound-healing and transwell assays were used to study the effects of TSN on glioma cell migration. The results of wound healing showed that the mobility of U-138MG and U-251MG cells was significantly decreased after TSN treatment (Figure 3A). As shown in Figure 3B, the 
invaded glioma cell numbers with TSN treatment were markedly reduced. In addition, the Western blot data showed that E-cadherin expression was up-regulated and $\mathrm{N}$-cadherin level was reduced after TSN treatment (Figure 3C). These data demonstrated that TSN treatment could suppress cell migration in U-138MG or U-251MG cells.

\section{TSN Increased miR-608 Level and Then Down-Regulate NotchI and Notch2 Expressions}

As indicated above, TSN treatment affected cell proliferation, apoptosis and migration in glioma cells. Nevertheless, the regulatory mechanism is still unknown. As shown in Figure 4A, TSN treatment significantly up-regulated miR-608 levels in U-138MG or U-251MG cells in a dose-dependent manner. In addition, Figure 4B demonstrated that Notch1 and Notch2 protein levels were markedly reduced after TSN treatment. Since TSN's regulation of miR-608 and Notch1 (Notch2) is in the opposite direction, there may exist a regulatory relationship between miR-608 and Notch1/Notch2.

By using TargetScan, we predicted that Notch1 and Notch2 are probably potential miR-608's target genes. Accordingly, the binding sites between miR-608 and Notch1/Notch2 3'-UTR are depicted in Figure 4C. The qRT-PCR results in Figure 4D demonstrated that miR608 level was markedly increased after transfected with miR-608 mimic, and decreased after transfected with miR608 inhibitors. As shown in Figure 4E, miR-608 upregulation reduced the luciferase activity of the Notch1 and Notch2 3'-UTR reporter plasmids. miR-608 inhibitors up-regulated the luciferase activity of the Notch1 and Notch2 3'-UTR reporter plasmids. However, the mutation of miR-608's binding sites within Notch1 and Notch2 3'UTR eliminated the significant alteration of the luciferase activities. The results of Western blot (Figure 4F) showed that the Notch 1 and Notch 2 protein levels were markedly decreased after transfected with miR-608 mimics.

A
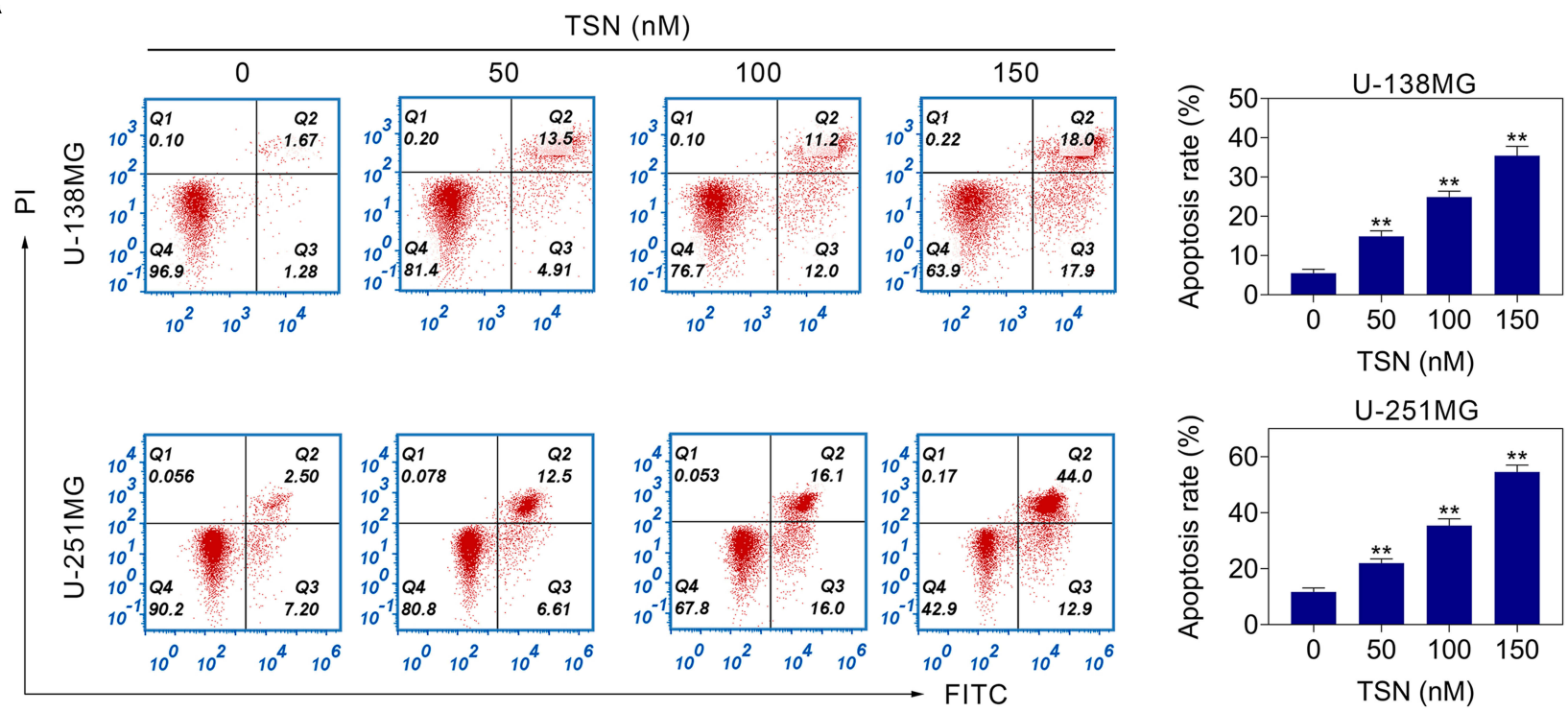

B
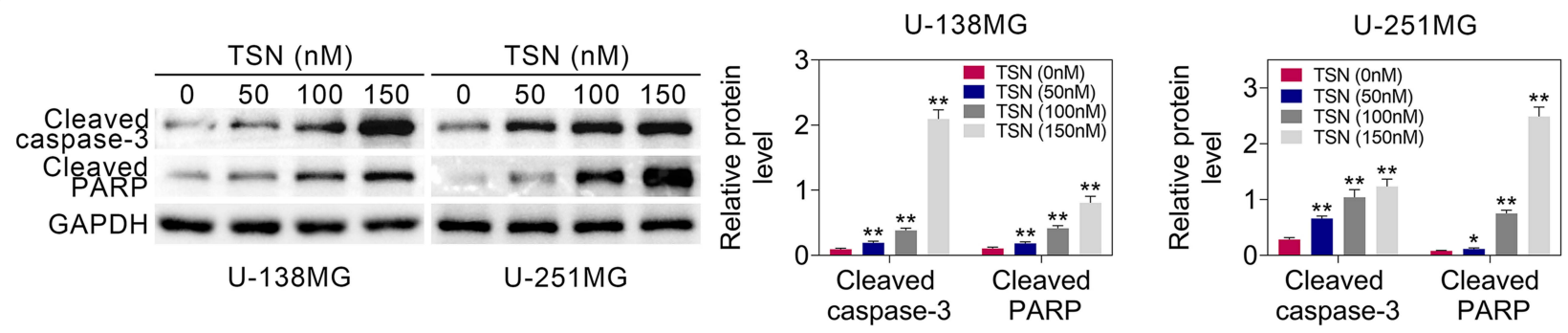

Figure 2 TSN affects the apoptosis of glioma cells. (A) Cell apoptosis analysis of U-I38MG or U-25IMG cells after TSN treatment (0nM, 50nM, I00nM, I50nM). Apoptosis rates were quantified and shown as histograms. (B) The protein levels of Cleaved caspase-3 and Cleaved PARP in U-I38MG or U-25IMG cells after TSN treatment (OnM, $50 \mathrm{nM}, 100 \mathrm{nM}, 150 \mathrm{nM})$, as determined using Western blotting. Relatively, quantitative results were determined by Image and shown as histogram. The data are expressed as the mean \pm SEM, $* P<0.05$, $* * P<0.01$. 
A

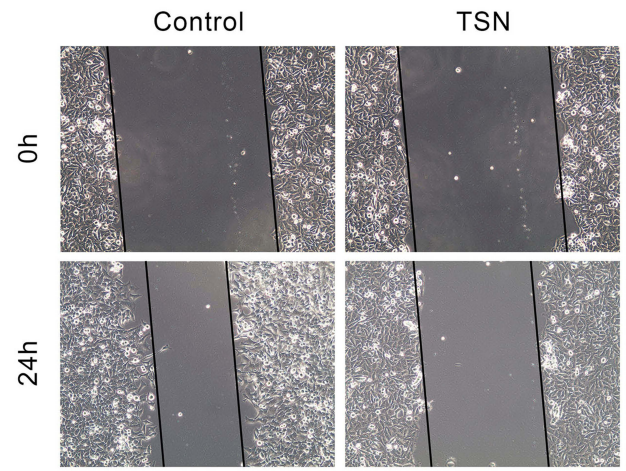

U-138MG

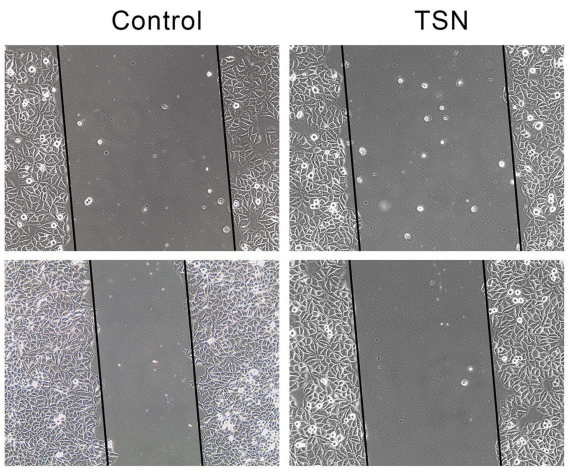

U-251MG

B
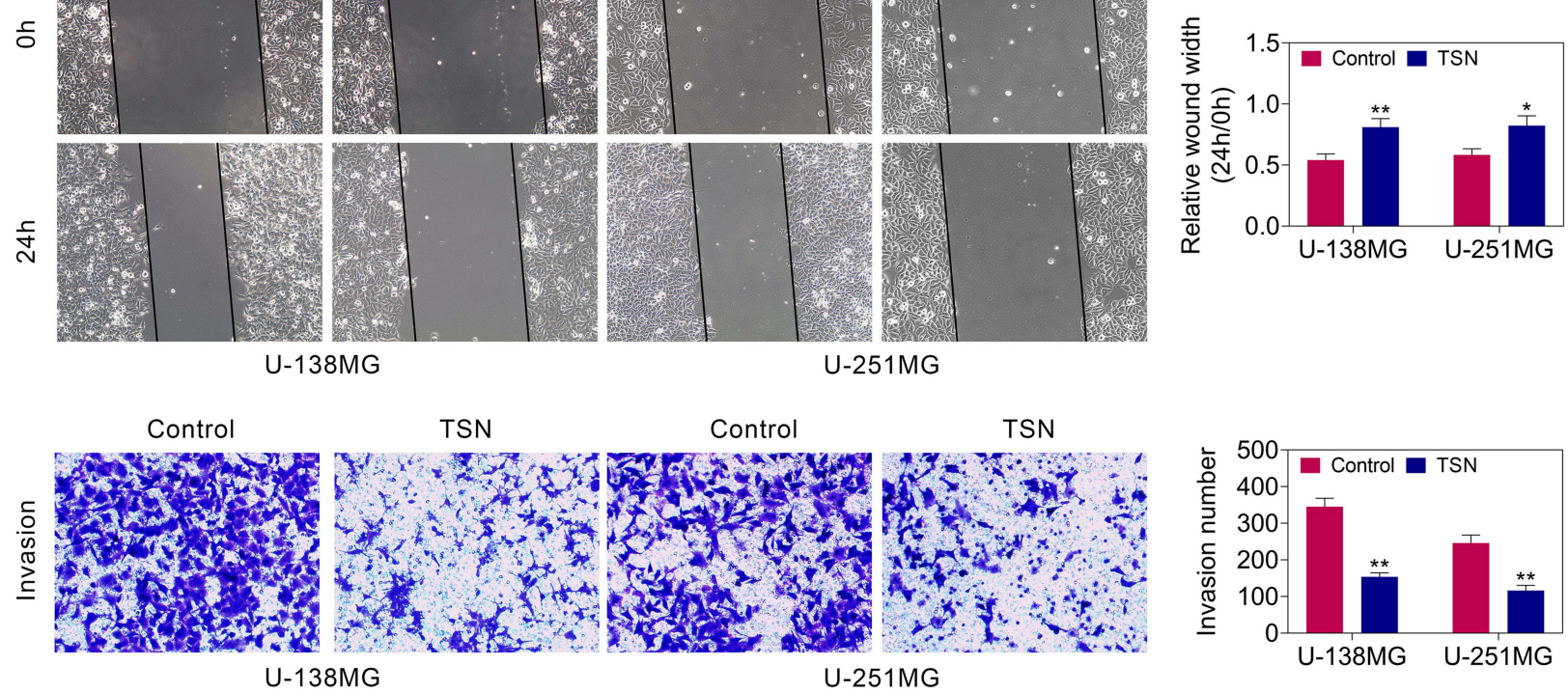

C

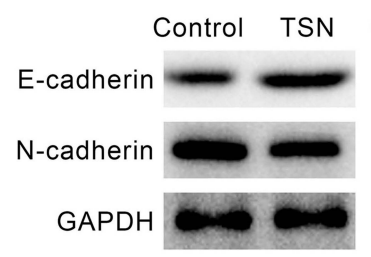

U-138MG

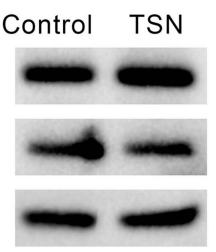

U-251MG

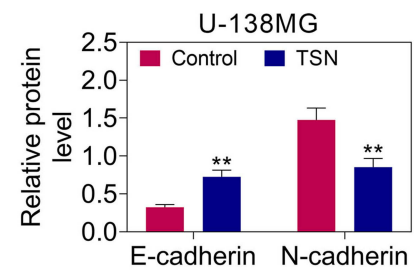

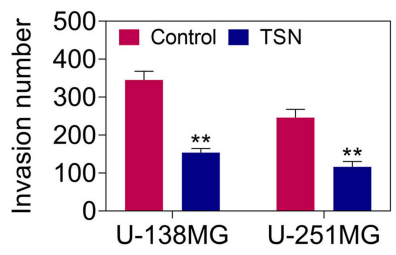

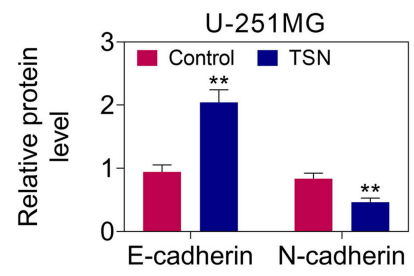

Figure 3 TSN effects the migration of glioma cells. (A) Wound scratch healing assay of U-I38MG or U-25IMG cells after TSN treatment (0nM, 50nM, I00nM, I50nM). Quantification of the wound-healing assay was shown as histograms. (B) Representative invasion assay images of U-I38MG or U-25IMG cells after TSN treatment (OnM, $50 \mathrm{nM}, 100 \mathrm{nM}, 150 \mathrm{nM}$ ). The invaded cells were quantified and shown as histograms. (C) The protein levels of E-cadherin and N-cadherin in U-I38MG or U-25IMG after TSN treatment $(0 \mathrm{nM}, 50 \mathrm{nM}, 100 \mathrm{nM}, 150 \mathrm{nM})$, as determined using Western blotting. Relatively quantitative results were determined by Image J and shown as histogram. The data are expressed as the mean \pm SEM, $* P<0.05$, **P $<0.0$ I.

Moreover, TSN-induced down-regulation of Notch1 and Notch2 was attenuated by the simultaneous treatment of TSN and miR-608 inhibitors (Figure 4G). These data indicated that TSN may suppress Notch1 and Notch2 expression through up-regulating miR-608.

\section{TSN Affects the Proliferation, Apoptosis and Migration of Glioma Cells By Regulating Notch I and Notch2}

To study whether TSN's influence on the behaviors of glioma cells was dependent on Notch1 and Notch2, expression plasmids of Notch1 and Notch2 were constructed. As shown in Figure 5A and B, mRNA and protein levels were significantly up-regulated in U-138MG cells after over-expression of Notch1 or Notch2. As the results of cell survival and cell colony formation assays revealed that the cell proliferation in glioma cells was suppressed after TSN treatment. Nevertheless, the effect was attenuated after simultaneously over-expressing Notch1 or Notch2 (Figure 5C and D). Cell apoptosis analysis demonstrated that TSNinduced up-regulation of apoptotic rate was decreased after over-expressing Notch1 or Notch2 (Figure 5E). Furthermore, the results of wound-healing and transwell assays showed that TSN treatment significantly reduced the migration and invasion of U-138MG and U-251MG cells. While, after over-expressing Notch1 or Notch2 simultaneously, the changes of cell invasion and cell migration induced by TSN were abolished (Figure 5F and G). These data together indicated that TSN treatment can affect the biological behaviors of glioma cells, including cell proliferation, cell apoptosis and cell migration, via negative regulation of Notch1 and Notch2. 
A

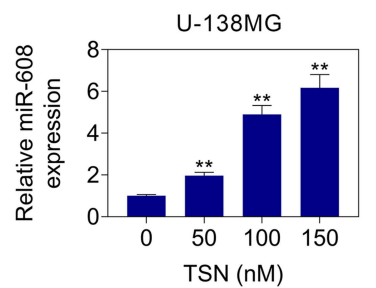

B
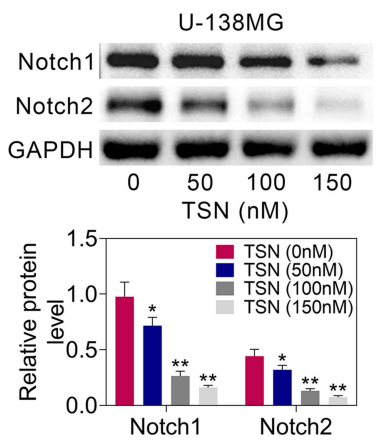

C

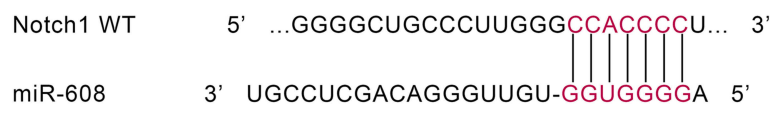

Notch1 MUT 5' ...GGGGCUGCCCUUGGGGGUGGGGU... 3'

Notch2 WT

$\operatorname{miR}-608$

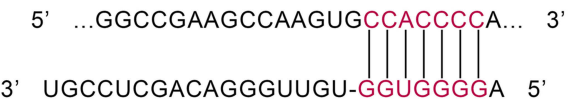

Notch2 MUT

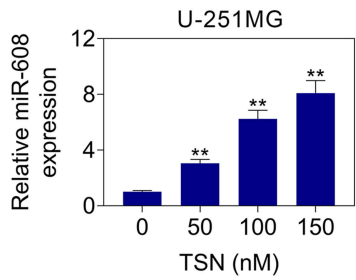

$\mathrm{U}-251 \mathrm{MG}$
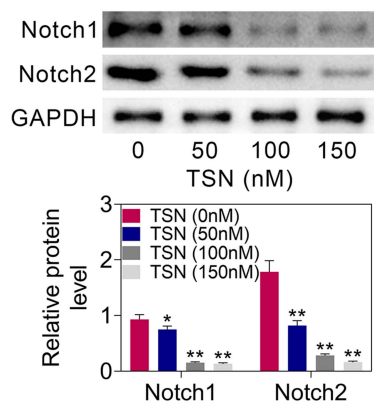

$\mathbf{F}$

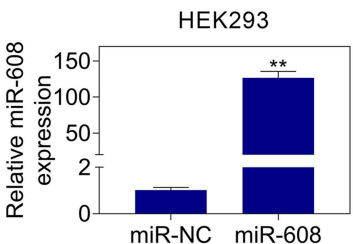

E
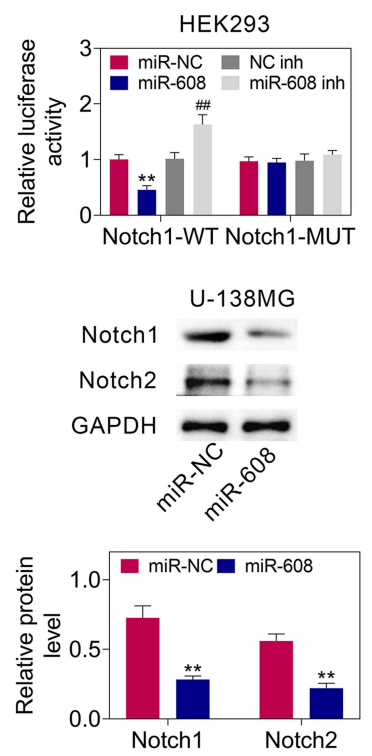

$\mathbf{G}$

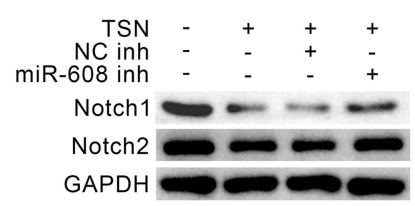

$\mathrm{U}-138 \mathrm{MG}$

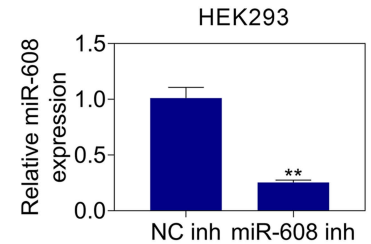

HEK293

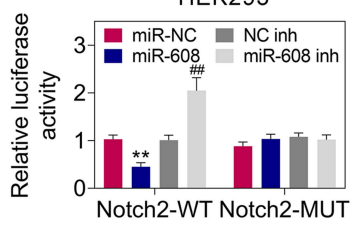

$\mathrm{U}-251 \mathrm{MG}$
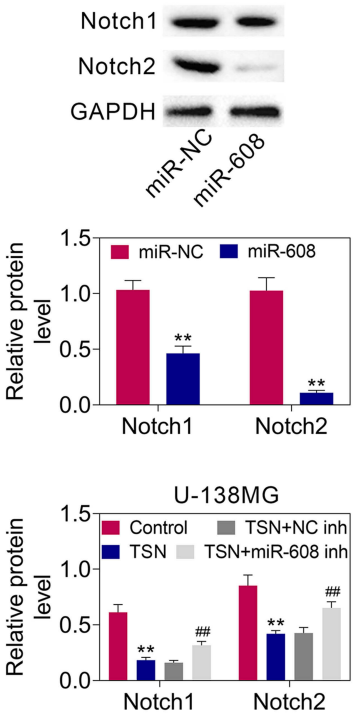

Figure 4 TSN up-regulates miR-608 level and negatively regulates NotchI and Notch2 expressions. (A) The relative miR-608 levels in U-I38MG or U-25IMG cells after TSN treatment (0nM, 50nM, 100nM, I50nM), as determined using qRT-PCR. (B) The protein levels of NotchI and Notch2 in U-I38MG or U-25IMG after TSN treatment $(0 \mathrm{nM}, 50 \mathrm{nM}, 100 \mathrm{nM}, 150 \mathrm{nM})$, as determined using Western blotting. Relatively quantitative results were determined by Image J and shown as histogram. (C) Intersection analysis of miR-608 and Notch I/Notch2 using prediction software of TargetScan. The predicted seed-recognition sites in the 3'-UTR of Notch I and Notch2 sequence and the corresponding miR-608 sequence was depicted. (D) The relative miR-608 levels in HEK293 cells after transfection with miR-NC or miR-608, as determined using qRTPCR. (E) The relative luciferase activity of the Notch I and Notch2 3'-UTR reporter plasmids was assayed in HEK293 cells after transfection with miR-NC, miR-608, NC-inh or miR-608-inh. The mutant NotchI and Notch2 3'-UTR reporters were also used as controls. (F) The protein levels of NotchI and Notch2 in U-I38MG or U-25IMG cells after transfection with miR-NC or miR-608, as determined using Western blotting. Relatively quantitative results were determined by Image J and shown as histogram. (G) The protein levels of Notchl and Notch2 in U-I38MG cells after treated with TSN, TSN+NC-inh or TSN+miR-608-inh, as determined using Western blotting. Relatively quantitative results were determined by ImageJ and shown as histogram. The data are expressed as the mean \pm SEM, ${ }^{* * / \# \#} P<0.01$.

\section{TSN Treatment Suppresses Tumor}

\section{Growth in a Mouse Xenograft Model}

Since TSN treatment can suppress cell proliferation, cell migration and promote cell apoptosis, its curative effect on glioma was next analyzed in vivo. The mouse xenograft tumor model was firstly constructed through subcutaneously implanting U-138MG cells into nude mice. Then, mice were randomly divided into two groups, TSN group and NC group. Mice of TSN group received i.p. injection of TSN ( $1 \mathrm{mg} / \mathrm{kg}$ per 2 days), and the mice of $\mathrm{NC}$ group were injected with the corresponding solvent DMSO. Twenty-eight days post-implantation, relevant tumors were excised and the representative image is presented in Figure 6A. The tumors in the TSN group exhibited smaller sizes and lighter weight, compared with those of the $\mathrm{NC}$ group. There was no statistically significant difference in the body weight between $\mathrm{NC}$ group mice and TSN group mice (Figure $6 \mathrm{~B})$, suggesting that the administration of TSN had no significant toxicity to mice. In addition, TSN treatment increased miR-608 level in xenograft tumors (Figure 6C). Moreover, TSN treatment down-regulated Ki67 positive cell number and Notch1/Notch2 protein levels (Figure 6D), and upregulated TUNEL positive cell percentage (Figure 6E) in 
A

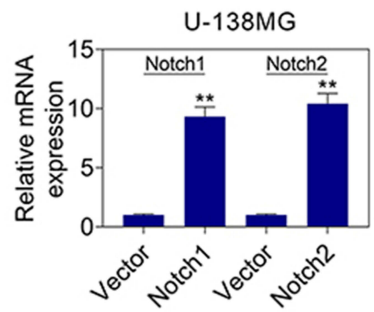

B

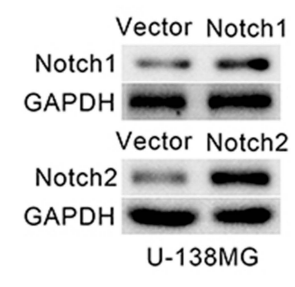

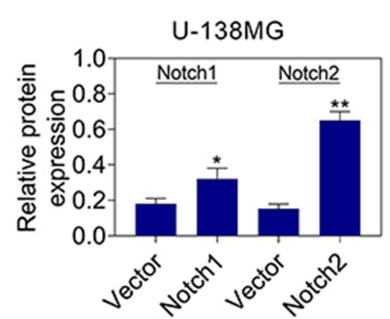

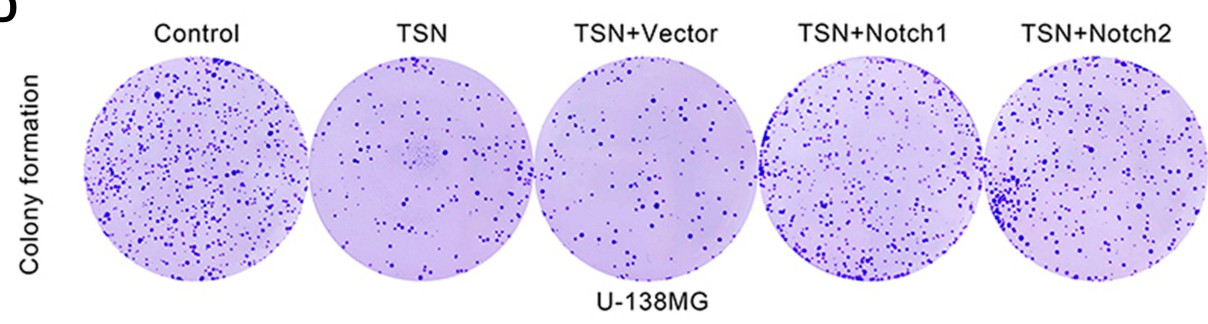

U-138MG

TSN+Vector

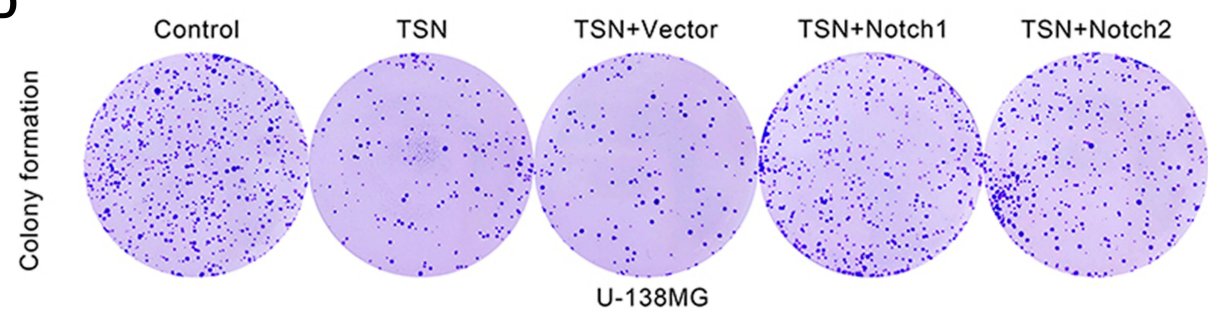

E

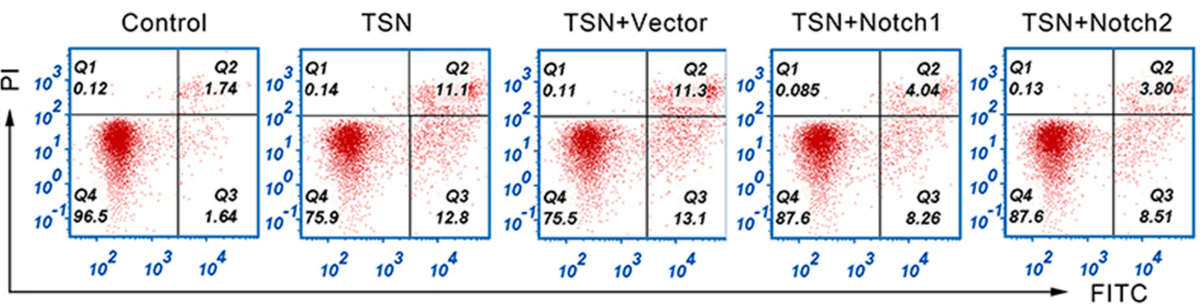

U-138MG

F

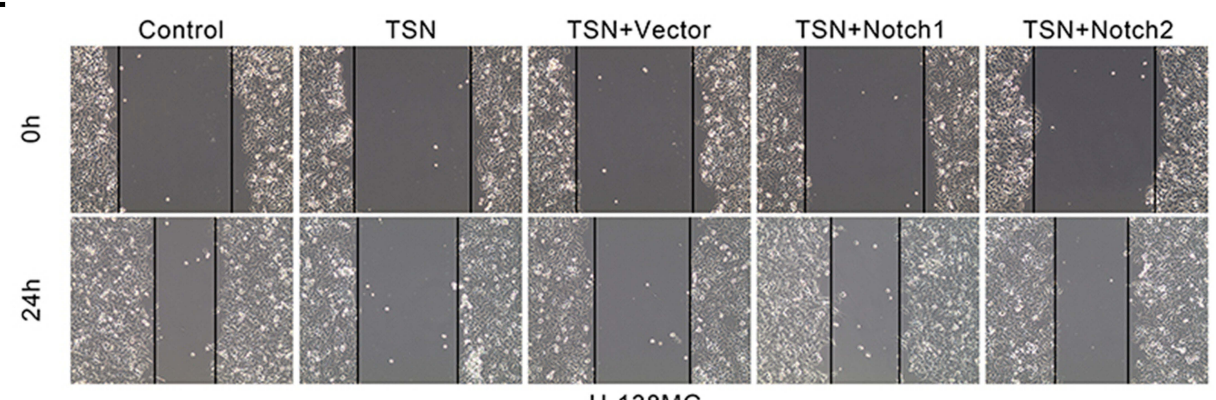

U-138MG

G

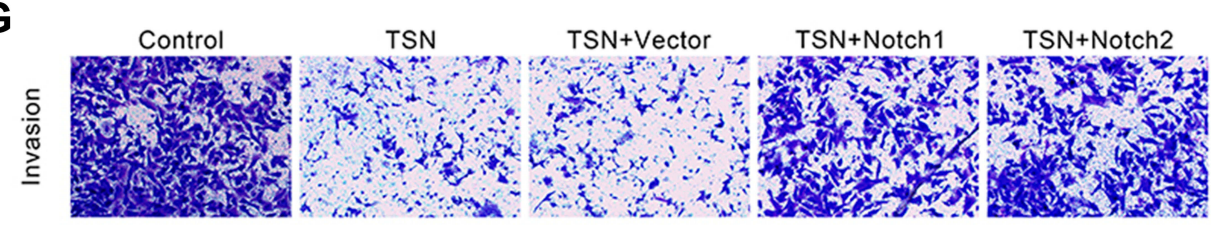

U-138MG

C
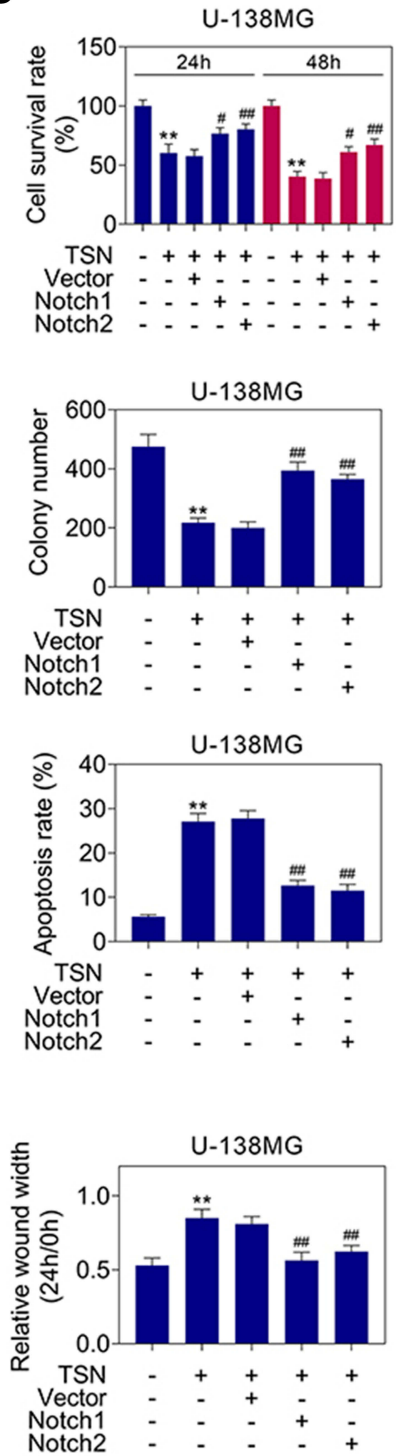

U-138MG

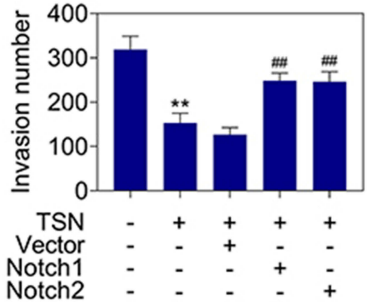

Figure 5 Notchl and Notch2 over-expression weakens TSN's effect on glioma cells. (A) The relative mRNA levels of Notch I or Notch2 in U-I38MG cells after transfected Notch I or Notch2 expressing plasmids, as determined using qRT-PCR. (B) The protein levels of Notch I and Notch2 in U-I38MG cells after NotchI or Notch2 expressing plasmids, as determined using Western blotting. Relatively quantitative results were determined by Image J and shown as histogram. (C) Cell survival of U-I38MG cells at $24 \mathrm{~h}$ and $48 \mathrm{~h}$ after treated with TSN, TSN+Vector, TSN+Notchl or TSN+Notch2. The measurements of the cell growth rate were obtained using a CCK-8 kit. (D) Colony formation analysis of U-I38MG cells after treated with TSN, TSN+Vector, TSN+Notchl or TSN+Notch2. Colony numbers were quantified and shown as histograms. (E) Cell apoptosis analysis of U-I38MG cells after treated with TSN, TSN+Vector, TSN+NotchI or TSN+Notch2. Apoptosis rates were quantified and shown as histograms. (F) Wound scratch healing assay of U-I38MG cells after treated with TSN, TSN+Vector, TSN+NotchI or TSN+Notch2. Quantification of the wound-healing assay was shown as histograms. (G) Representative invasion assay images of U-I38MG cells after treated with TSN, TSN+Vector, TSN+NotchI or TSN+Notch2. The invaded cells were quantified and shown as histograms. The data are expressed as the mean $\pm \mathrm{SEM},{ }^{* / \#} P<0.0 \mathrm{I},{ }^{* * / \# \#} P<0.0 \mathrm{I}$. 
tumor tissues. These results indicated that TSN treatment can increase miR-608 level, down-regulate the protein expressions of Notch1 and Notch2, and finally suppress the glioma growth in vivo.

\section{Discussion}

In the present study, we not only investigated the biological effects mediated by TSN treatment on cell proliferation, apoptosis and migration, but also demonstrated its functional mechanism via regulation of miR-608/Notch1 (Notch2) axis (Figure 7). As a natural molecular product, TSN possesses the characteristics of multiple targets. For instance, TSN was reported to block STAT3 dimerization and impair the complex formation of STAT3 and epidermal growth factor receptor (EGFR) in osteosarcoma. ${ }^{11}$ To a large extent, these functional ways above are direct mode of action. Recently, it was reported that TSN was associated with noncoding RNA expression, which indirectly exerts regulation function. Lu et al demonstrated that integrated miRNA-mRNA approach can be used as a tool to better understand the complex and dynamic behavior of toosendanin-induced liver injury in mice. ${ }^{30}$ Circulating miR-122 and miR-192 have emerged as promising biomarkers of liver injury in drug-induced liver injury patients. ${ }^{31}$ Furthermore, TSN can increase the level of miR-200a and then mediate suppressive effects of $\beta$-catenin pathway and suppress oncogenic phenotypes of gastric carcinoma cells. ${ }^{18}$
Previous studies indicated that miR-200a was down-regulated in glioma and was able to affect cell growth and migration by targeting G protein $\alpha$ inhibitory subunit 1 (Gail) and singleminded homolog 2-short form (SIM2-s). ${ }^{32,33}$ Thus, the effect of TSN on miR-200a in glioma needs to be further demonstrated. It can be hypothesized that TSN may affect glioma development by simultaneously regulating several miRNAs, including miR-608 and miR-200a.

Next, we explored the miR-608's potential target. By using Targetscan prediction, potential seed binding sites between miR-608 and Notch1 and Notch2 3'-UTR were predicted. Except for Notch1 and Notch2, macrophage migration inhibitory factor was found to be targeted by miR-608 in glioma so far. ${ }^{23}$ Moreover, some tumor-associated genes, mainly including ribonucleotide reductase M1 (RRM1) in pancreatic cancer, ${ }^{34}$ frizzled family receptor 4 (FZD4) in ovarian cancer, ${ }^{35}$ nucleus accumbens-associated protein 1 (NACC1) in melanoma, ${ }^{36}$ were reported to be targeted by miR- $608 .{ }^{37}$ Since one miRNA could regulate multi-genes, it is worth to further verify whether miR-608 can negatively regulate these genes in glioma. Thus, we speculated that miR-608 might act as a heeler of TSN, which can be up-regulated by TSN and regulate its target genes during glioma development.

As an important mediator of cancer-related signaling, Notch signaling was an evolutionarily conserved and demonstrated to be associated with glioma. ${ }^{38}$ Our
A

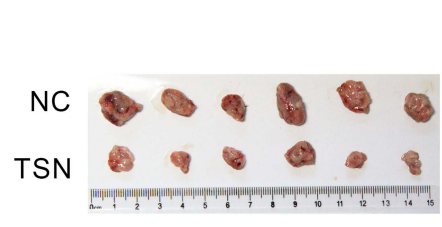

C

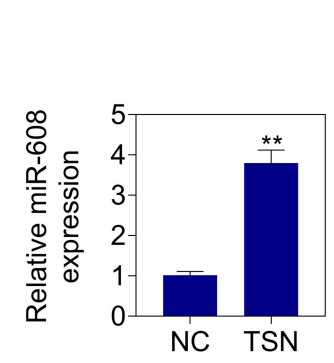

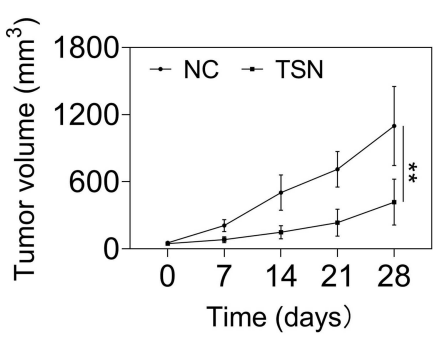

D

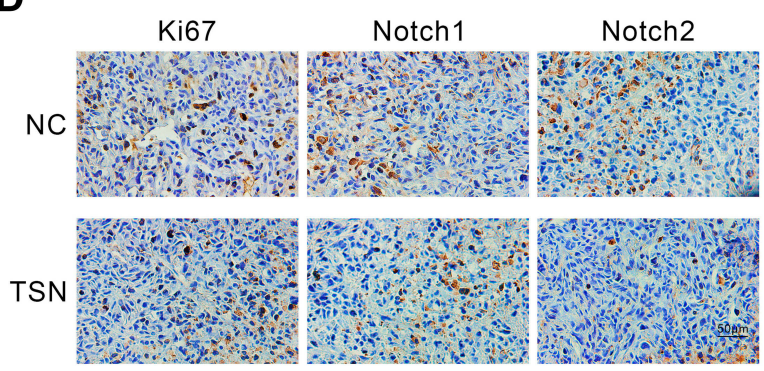

B
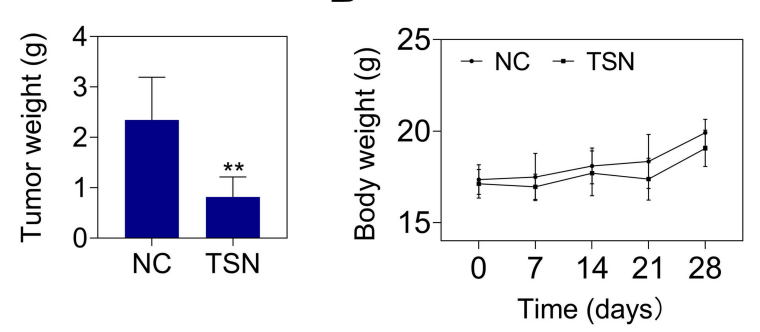

E

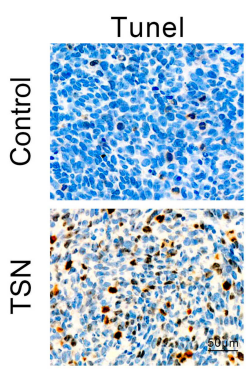

Figure 6 TSN treatment inhibits tumor growth in a glioma mouse xenograft model. (A) Representative photograph of corresponding tumors dissected from mice at day 28 post-implantation (left). The xenograft tumor volumes (middle) and weight (right) of nude mice derived from subcutaneous implantation of $U$ - I $38 \mathrm{MG}$ cells. (B) The body weight of mice from NC or TSN groups. (C) The relative miR-608 level in the xenograft tumors from NC or TSN groups. (D) IHC staining of Ki67 and Notch I/Notch2 in the xenograft tumors from NC or TSN groups. (E) TUNEL analysis in the xenograft tumors from NC or TSN groups. The data are expressed as the mean \pm SEM, $* * P<0.0$ I. 


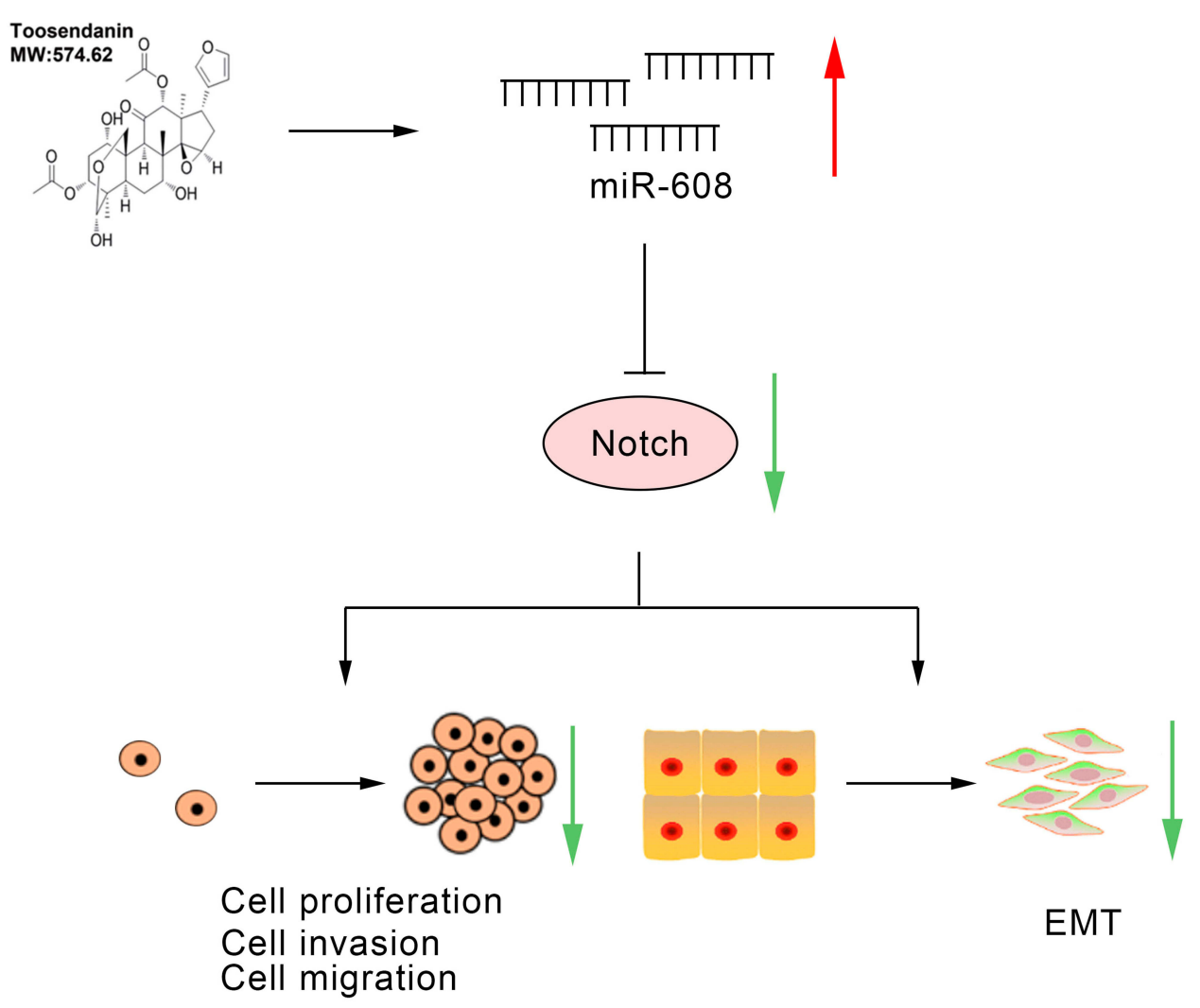

Figure 7 Schematic diagram of the proposed mechanism. miR-203 expression may suppress glioma progression property and induces apoptosis by up-regulating miR-608 and inhibiting Notch 1 and Notch2.

data in this study showed that over-expression of Notch1 and Notch2 could promote glioma cell proliferation and migration, which was inhibited by TSNincreased miR-608. Therefore, Notch1 and Notch2 stand out as particular targets for glioma prevention. In addition to miR-608, only miR-34a was found to simultaneously regulate Notch1 and Notch2 in glioma development. ${ }^{26}$ Since inhibition of one member of the Notch family can selectively suppress the growth of glioma, these vital miRNAs of miR-608 and miR-34a seem to be very powerful due to their inhibitory effects on both Notch1 and Notch2. Some other miRNAs were demonstrated to target Notch1 or Notch2 in glioma. Among them, miR-499a and miR-139-5p could inhibit Notch1 expression in glioma. ${ }^{39,40}$ And miR-181a and miR-107 are also identified as important posttranscriptional regulators of Notch2 in glioma. ${ }^{41,42}$ These findings not only contribute to understand the molecular mechanism of Notch1 and Notch2 dysregulation in glioma, but also provide possible targets for glioma intervention. Moreover, some other Notch1 or Notch2 inhibitors, such as $\gamma$-secretase inhibitors, are also clinically promising. ${ }^{43}$ Because of the inevitable limitations of one kind drug, combined utilization of multi-drugs, such as TSN, miRNAs or specific antibody, might prove the potential application for glioma therapy.

In summary, our data demonstrated that TSN treatment can inhibit cell proliferation, cell migration and promote cell apoptosis in glioma cells. TSN could up-regulate miR-608 and decrease the expression level of its targets, Notch1 and Notch2. Over-expression of Notch1 or Notch2 could restore the TSN-induced tumor-suppressive effects. Additionally, TSN can inhibit tumor growth by up-regulation of miR-608 and then inhibiting the expressions of Notch1 and Notch2 in vivo. Based on the biological effects on tumor progression, the molecular axis of TSN/miR-608/Notch1 (Notch2) has the potential to become promising candidates for glioma intervention.

\section{Ethics and Consent Statement}

All experiments with nude mice were constructed in accordance with the Guidelines for the Care and Use of Laboratory Animals, which was presented by the National 
Institutes of Health and approved by the Ethics Committee of Zhejiang Cancer Hospital (Approval no.2019-05-014).

\section{Author Contributions}

$\mathrm{PH}$ conceived and designed the experiments, QW analyzed and interpreted the results of the experiments, ZW and GLH performed the experiments. All authors contributed to data analysis, drafting or revising the article, gave final approval of the version to be published, and agree to be accountable for all aspects of the work.

\section{Funding}

This work was supported by Zhejiang Provincial National Science Foundation of China. (Grant No. YQ20H280002, Q18H290004).

\section{Disclosure}

The authors state that there are no conflicts of interest to disclose.

\section{References}

1. Jemal A, Bray F, Center MM, Ferlay J, Ward E, Forman D. Global cancer statistics. CA Cancer J Clin. 2011;61(2):69-90. doi:10.3322/ caac. 20107

2. Milano MT, Johnson MD, Sul J, et al. Primary spinal cord glioma: a surveillance, epidemiology, and end results database study. $J$ Neurooncol. 2010;98(1):83-92. doi:10.1007/s11060-009-0054-7

3. Louis DN, Perry A, Reifenberger G, et al. The 2016 World Health Organization classification of tumors of the central nervous system: a summary. Acta Neuropathol. 2016;131(6):803-820. doi:10.1007/ s00401-016-1545-1

4. Ohgaki H, Kleihues P. Population-based studies on incidence, survival rates, and genetic alterations in astrocytic and oligodendroglial gliomas. J Neuropathol Exp Neurol. 2005;64(6):479-489. doi:10.1093/jnen/ 64.6.479

5. Bleeker FE, Molenaar RJ, Leenstra S. Recent advances in the molecular understanding of glioblastoma. $J$ Neurooncol. 2012;108 (1):11-27. doi:10.1007/s11060-011-0793-0

6. Wang Y, Jiang T. Understanding high grade glioma: molecular mechanism, therapy and comprehensive management. Cancer Lett. 2013;331(2):139-146. doi:10.1016/j.canlet.2012.12.024

7. Muhammad N, Steele R, Isbell TS, Philips N, Ray RB. Bitter melon extract inhibits breast cancer growth in preclinical model by inducing autophagic cell death. Oncotarget. 2017;8(39):66226-66236. doi:10.18632/oncotarget.19887

8. Bhattacharya S, Muhammad N, Steele R, Kornbluth J, Ray RB. Bitter melon enhances natural killer-mediated toxicity against head and neck cancer cells. Cancer Prev Res (Phila). 2017;10(6):337-344. doi:10.1158/1940-6207.CAPR-17-0046

9. Bhattacharya S, Muhammad N, Steele R, Peng G, Ray RB. Immunomodulatory role of bitter melon extract in inhibition of head and neck squamous cell carcinoma growth. Oncotarget. 2016;7(22):33202-33209. doi:10.18632/oncotarget.8898

10. Zhang B, Wang ZF, Tang MZ, Shi YL. Growth inhibition and apoptosis-induced effect on human cancer cells of toosendanin, a triterpenoid derivative from chinese traditional medicine. Invest New Drugs. 2005;23(6):547-553. doi:10.1007/s10637-005-0909-5
11. Zhang $\mathrm{T}$, Li J, Yin F, et al. Toosendanin demonstrates promising antitumor efficacy in osteosarcoma by targeting STAT3. Oncogene. 2017;36(47):6627-6639. doi:10.1038/onc.2017.270

12. Pei Z, Fu W, Wang G. A natural product toosendanin inhibits epithelial-mesenchymal transition and tumor growth in pancreatic cancer via deactivating Akt/mTOR signaling. Biochem Biophys Res Commun. 2017;493(1):455-460. doi:10.1016/j.bbrc.2017.08.170

13. Zhou Q, Wu X, Wen C, et al. Toosendanin induces caspase-dependent apoptosis through the p38 MAPK pathway in human gastric cancer cells. Biochem Biophys Res Commun. 2018;505(1):261-266. doi:10.1016/j.bbrc.2018.09.093

14. Cao L, Qu D, Wang H, et al. Toosendanin exerts an anti-cancer effect in glioblastoma by inducing estrogen receptor beta- and p53-mediated apoptosis. Int J Mol Sci. 2016;17(11):1928. doi:10.3390/ijms17111928

15. Rupaimoole R, Slack FJ. MicroRNA therapeutics: towards a new era for the management of cancer and other diseases. Nat Rev Drug Discov. 2017;16(3):203-222. doi:10.1038/nrd.2016.246

16. Muhammad N, Bhattacharya S, Steele R, Ray RB. Anti-miR-203 suppresses ER-positive breast cancer growth and stemness by targeting SOCS3. Oncotarget. 2016;7(36):58595-58605. doi:10.18632/ oncotarget.11193

17. Jiang Y, Zhou J, Zhao J, et al. MiR-18a-downregulated RORA inhibits the proliferation and tumorigenesis of glioma using the TNF-alpha-mediated NF-kappaB signaling pathway. EBioMedicine. 2020;52:102651. doi:10.1016/j.ebiom.2020.102651

18. Wang G, Huang YX, Zhang R, et al. Toosendanin suppresses oncogenic phenotypes of human gastric carcinoma SGC7901 cells partly via miR200amediated downregulation of beta-catenin pathway. Int J Oncol. 2017;51(5):1563-1573. doi:10.3892/ijo.2017.4139

19. He L, Meng D, Zhang SH, Zhang Y, Deng Z, Kong LB. microRNA608 inhibits human hepatocellular carcinoma cell proliferation via targeting the BET family protein BRD4. Biochem Biophys Res Commun. 2018;501(4):1060-1067. doi:10.1016/j.bbrc.2018.05.108

20. Yang H, Li Q, Niu J, et al. microRNA-342-5p and miR-608 inhibit colon cancer tumorigenesis by targeting NAA10. Oncotarget. 2016;7 (3):2709-2720. doi:10.18632/oncotarget.6458

21. Yu HX, Wang XM, Han XD, Cao BF. MiR-608 exerts tumor suppressive function in lung adenocarcinoma by directly targeting MIF. Eur Rev Med Pharmacol Sci. 2018;22(15):4908-4916. doi:10.26355/ eurrev_201808_15629

22. Liang Z, Wang X, Xu X, et al. MicroRNA-608 inhibits proliferation of bladder cancer via AKT/FOXO3a signaling pathway. Mol Cancer. 2017;16(1):96. doi:10.1186/s12943-017-0664-1

23. Wang Z, Xue Y, Wang P, Zhu J, Ma J. MiR-608 inhibits the migration and invasion of glioma stem cells by targeting macrophage migration inhibitory factor. Oncol Rep. 2016;35(5):2733-2742. doi:10.3892/ or.2016.4652

24. Li J, Cui Y, Gao G, Zhao Z, Zhang H, Wang X. Notch1 is an independent prognostic factor for patients with glioma. J Surg Oncol. 2011;103(8):813-817. doi:10.1002/jso.21851

25. Yu HP, Qi ST, Feng WF, Zhang GZ, Zhang HP, Tian JJ. Interference of Notch 2 inhibits the progression of gliomas and induces cell apoptosis by induction of the cell cycle at the G0/G1 phase. Mol Med Rep. 2015;11(1):734-738. doi:10.3892/mmr.2014.2747

26. Li Y, Guessous F, Zhang Y, et al. MicroRNA-34a inhibits glioblastoma growth by targeting multiple oncogenes. Cancer Res. 2009;69 (19):7569-7576. doi:10.1158/0008-5472.CAN-09-0529

27. Kefas B, Comeau L, Floyd DH, et al. The neuronal microRNA miR-326 acts in a feedback loop with notch and has therapeutic potential against brain tumors. J Neurosci. 2009;29 (48):15161-15168. doi:10.1523/JNEUROSCI.4966-09.2009

28. Hu Y, Liu Q, Zhang M, Yan Y, Yu H, Ge L. MicroRNA-362-3p attenuates motor deficit following spinal cord injury via targeting paired box gene 2. J Integr Neurosci. 2019;18(1):57-64. doi:10.31083/j.jin.2019.01.12 
29. Guide for the Care and Use of Laboratory Animals. The National Academies Collection: Reports Funded by National Institutes of Health. Washington (DC); 2011.

30. Lu X, Ji C, Tong W, et al. Integrated analysis of microRNA and mRNA expression profiles highlights the complex and dynamic behavior of toosendanin-induced liver injury in mice. Sci Rep. 2016;6(1):34225. doi:10.1038/srep34225

31. Krauskopf J, Caiment F, Claessen SM, et al. Application of high-throughput sequencing to circulating microRNAs reveals novel biomarkers for drug-induced liver injury. Toxicol Sci. 2015;143 (2):268-276. doi:10.1093/toxsci/kfu232

32. Liu YY, Chen MB, Cheng L, et al. microRNA-200a downregulation in human glioma leads to Galphail over-expression, Akt activation, and cell proliferation. Oncogene. 2018;37(21):2890-2902. doi:10.1038/s41388-018-0184-5

33. Su Y, He Q, Deng L, et al. MiR-200a impairs glioma cell growth, migration, and invasion by targeting SIM2-s. Neuroreport. 2014;25 (1):12-17. doi:10.1097/WNR.0000000000000032

34. Rajabpour A, Afgar A, Mahmoodzadeh H, Radfar JE, Rajaei F, Teimoori-Toolabi L. MiR-608 regulating the expression of ribonucleotide reductase M1 and cytidine deaminase is repressed through induced gemcitabine chemoresistance in pancreatic cancer cells. Cancer Chemother Pharmacol. 2017;80(4):765-775. doi:10.1007/ s00280-017-3418-2

35. Wang Y, Zhang W, Wang Y, Wang S. HOXD-AS1 promotes cell proliferation, migration and invasion through miR-608/FZD4 axis in ovarian cancer. Am J Cancer Res. 2018;8(1):170-182.

36. Jiao H, Jiang S, Wang H, Li Y, Zhang W. Upregulation of LINC00963 facilitates melanoma progression through miR-608/ NACC1 pathway and predicts poor prognosis. Biochem Biophys Res Commun. 2018;504(1):34-39. doi:10.1016/j.bbrc.2018.08.115
37. Zhang Y, Schiff D, Park D, Abounader R, Mott JL. MicroRNA-608 and microRNA-34a regulate chordoma malignancy by targeting EGFR, Bcl-xL and MET. PLoS One. 2014;9(3):e91546. doi:10.1371/journal.pone.0091546

38. Yan D, Hao C, Xiao-Feng L, Yu-Chen L, Yu-Bin F, Lei Z. Molecular mechanism of Notch signaling with special emphasis on microRNAs: implications for glioma. J Cell Physiol. 2018;234(1):158-170. doi:10.1002/jcp.26775

39. Wang BQ, Yang B, Yang HC, et al. MicroRNA-499a decelerates glioma cell proliferation while accelerating apoptosis through the suppression of Notch1 and the MAPK signaling pathway. Brain Res Bull. 2018;142:96-106. doi:10.1016/j.brainresbull.2018.06.005

40. Li J, Li Q, Lin L, et al. Targeting the Notch1 oncogene by miR-139$5 \mathrm{p}$ inhibits glioma metastasis and epithelial-mesenchymal transition (EMT). BMC Neurol. 2018;18(1):133. doi:10.1186/s12883-018$1139-8$

41. Huang SX, Zhao ZY, Weng GH, et al. Upregulation of miR-181a suppresses the formation of glioblastoma stem cells by targeting the Notch2 oncogene and correlates with good prognosis in patients with glioblastoma multiforme. Biochem Biophys Res Commun. 2017;486 (4):1129-1136. doi:10.1016/j.bbrc.2017.04.008

42. Chen L, Chen XR, Zhang R, et al. MicroRNA-107 inhibits glioma cell migration and invasion by modulating Notch2 expression. $J$ Neurooncol. 2013;112(1):59-66. doi:10.1007/s11060-012-1037-7

43. Hiddingh L, Tannous BA, Teng $J$, et al. EFEMP1 induces gamma-secretase/Notch-mediated temozolomide resistance in glioblastoma. Oncotarget. 2014;5(2):363-374. doi:10.18632/ oncotarget. 1620
Cancer Management and Research

\section{Publish your work in this journal}

Cancer Management and Research is an international, peer-reviewed open access journal focusing on cancer research and the optimal use of preventative and integrated treatment interventions to achieve improved outcomes, enhanced survival and quality of life for the cancer patient.
The manuscript management system is completely online and includes a very quick and fair peer-review system, which is all easy to use Visit http://www.dovepress.com/testimonials.php to read real quotes from published authors. 